\title{
Dehn surgery, homology and hyperbolic volume
}

\author{
IAN AGOL \\ MARC CUller \\ Peter B Shalen
}

\begin{abstract}
If a closed, orientable hyperbolic 3-manifold $M$ has volume at most 1.22 then $H_{1}\left(M ; \mathbb{Z}_{p}\right)$ has dimension at most 2 for every prime $p \neq 2,7$, and $H_{1}\left(M ; \mathbb{Z}_{2}\right)$ and $H_{1}\left(M ; \mathbb{Z}_{7}\right)$ have dimension at most 3 . The proof combines several deep results about hyperbolic 3-manifolds. The strategy is to compare the volume of a tube about a shortest closed geodesic $C \subset M$ with the volumes of tubes about short closed geodesics in a sequence of hyperbolic manifolds obtained from $M$ by Dehn surgeries on $C$.
\end{abstract}

57M50; 57M27

\section{Introduction}

We shall prove:

Theorem 1.1 Suppose that $M$ is a closed, orientable hyperbolic 3-manifold with volume at most 1.22 . Then $H_{1}\left(M ; \mathbb{Z}_{p}\right)$ has dimension at most 2 for every prime $p \neq 2,7$, and $H_{1}\left(M ; \mathbb{Z}_{2}\right)$ and $H_{1}\left(M ; \mathbb{Z}_{7}\right)$ have dimension at most 3. Furthermore, if $M$ has volume at most 1.182 , then $H_{1}\left(M ; \mathbb{Z}_{7}\right)$ has dimension at most 2.

The bound of 2 for the dimension of $H_{1}\left(M ; \mathbb{Z}_{p}\right)$ is sharp when $p$ is 3 or 5 . Indeed, the manifolds $\mathrm{m} 003(-3,1)$, and $\mathrm{m} 007(3,1)$ from the list given in $[10]$ have respective volumes $0.94 \ldots$ and $1.01 \ldots$, and their integer homology groups are respectively isomorphic to $\mathbb{Z}_{5} \oplus \mathbb{Z}_{5}$ and $\mathbb{Z}_{3} \oplus \mathbb{Z}_{6}$.

Apart from these two examples, the only example known to us of a closed, orientable hyperbolic 3 -manifold with volume at most 1.22 is the manifold m003 $(-2,3)$ from the list given in [10]. These three examples suggest that the bounds for the dimension of $H_{1}\left(M ; \mathbb{Z}_{p}\right)$ given by Theorem 1.1 may not be sharp for $p \neq 3,5$.

The proof of Theorem 1.1 depends on several deep results, including a strong form of the "log 3 Theorem" of Anderson, Canary, Culler and Shalen [4; 8]; the Embedded Tube Theorem of Gabai, Meyerhoff and N Thurston [9]; the Marden Tameness Conjecture, 
recently proved by Agol [1] and by Calegari and Gabai [7]; and an even more recent result due to Agol, Dunfield, Storm and W Thurston [3]. The strategy of our proof is to compare the volume of a tube about a shortest closed geodesic $C \subset M$ with the volumes of tubes about short closed geodesics in a sequence of hyperbolic manifolds obtained from $M$ by Dehn surgeries on $C$.

After establishing some basic conventions in Section 2, we carry out the strategy described above in Sections 3-6, for the case of manifolds which are "non-exceptional" in the sense that they contain shortest geodesics with tube radius greater than $(\log 3) / 2$. In Section 5, for the case of non-exceptional manifolds with volume at most 1.22, we establish a bound of 3 for the dimension of $H_{1}\left(M ; \mathbb{Z}_{p}\right)$ for any prime $p$. In Section 6 , again for the case of non-exceptional manifolds with volume at most 1.22, we establish a bound of 2 for the dimension of $H_{1}\left(M ; \mathbb{Z}_{p}\right)$ for any odd prime $p$. In Section 7 we use results from [9] to handle the case of exceptional manifolds, and complete the proof of Theorem 1.1.

The research described in this paper was partially supported by NSF grants DMS0204142 and DMS-0504975.

\section{Definitions and conventions}

2.1 If $g$ is a loxodromic isometry of hyperbolic 3-space $\mathbb{U}^{3}$ we shall let $A_{g}$ denote the hyperbolic geodesic which is the axis of $g$. The cylinder about $A_{g}$ of radius $r$ is the open set $Z_{r}(g)=\left\{x \in \mathbb{Q}^{3} \mid \operatorname{dist}\left(x, A_{g}\right)<r\right\}$.

2.2 Suppose that $M$ is a complete, orientable hyperbolic 3-manifold. Let us identify $M$ with $\mathbb{H}^{3} / \Gamma$, where $\Gamma \cong \pi_{1}(M)$ is a discrete, torsion-free subgroup of Isom $+\mathbb{H}^{3}$. If $C$ is a simple closed geodesic in $M$ then there is a loxodromic isometry $g \in \Gamma$ with $A_{g} /\langle g\rangle=C$. For any $r>0$ the image $Z_{r}(g) /\langle g\rangle$ of $Z_{r}(g)$ under the covering projection is a neighborhood of $C$ in $M$. For sufficiently small $r>0$ we have

$$
\left\{h \in \Gamma \mid h\left(Z_{r}(g)\right) \cap Z_{r}(g) \neq \varnothing\right\}=\langle g\rangle .
$$

Let $R$ denote the supremum of the set of $r$ for which this condition holds. We define tube $(C)=Z_{R}(g) /\langle g\rangle$ to be the maximal tube about $C$. We shall refer to $R$ as the tube radius of $C$, and denote it by $\operatorname{tuberad}(C)$.

2.3 If $C$ is a simple closed geodesic in a closed hyperbolic 3-manifold $M$, it follows from [13], [2] that $M-C$ is homeomorphic to a hyperbolic manifold $N$ of finite volume having one cusp. The manifold $N$, which by Mostow rigidity is unique up to isometry, will be denoted $\operatorname{drill}_{C}(M)$. 
2.4 If $C$ is a shortest closed geodesic in a closed hyperbolic 3-manifold $M$, ie, one such that length $(C) \leq$ length $\left(C^{\prime}\right)$ for every other closed geodesic $C^{\prime}$, then in particular $C$ is simple, and the notions of 2.2 and 2.3 apply to $C$.

2.5 Suppose that $N=\mathbb{\boxplus}^{3} / \Gamma$ is a non-compact orientable complete hyperbolic manifold of finite volume. Let $\Pi \cong \mathbb{Z} \times \mathbb{Z}$ be a maximal parabolic subgroup of $\Gamma$ (so that $\Pi$ corresponds to a peripheral subgroup under the isomorphism of $\Gamma$ with $\left.\pi_{1}(N)\right)$. Let $\xi$ denote the fixed point of $\Pi$ on the sphere at infinity and let $B$ be an open horoball centered at $\xi$ such that $\{g \in \Gamma \mid g B \cap B \neq \varnothing\}=\Pi$. Then $\mathcal{H}=B / \Pi$, which we identify with the image of $B$ in $N$, is called a cusp neighborhood in $N$.

If $\mathcal{H}$ is a cusp neighborhood in $N=\mathbb{U}^{3} / \Gamma$ then the inverse image of $\mathcal{H}$ under the covering projection $\mathbb{M}^{3} \rightarrow N$ is a union of disjoint open horoballs. The cusp neighborhood $\mathcal{H}$ is maximal if and only there exist two of these disjoint horoballs whose closures have non-empty intersection.

2.6 If $N$ is a complete, orientable hyperbolic manifold of finite volume, $\hat{N}$ will denote a compact core of $N$. Thus $\hat{N}$ is a compact 3-manifold whose boundary components are all tori, and the number of these tori is equal to the number of cusps of $N$.

\section{Drilling and packing}

Lemma 3.1 Suppose that $M$ is a closed, orientable hyperbolic 3-manifold, and that $C$ is a shortest geodesic in $M$. Set $N=\operatorname{drill}_{C}(M)$. If tuberad $(C) \geq(\log 3) / 2$ then $\operatorname{vol} N<3.0177 \operatorname{vol} M$.

Proof The proof is based on a result due to Agol, Dunfield, Storm and W Thurston [3]. We let $L$ denote the length of the geodesic $C$ in the closed hyperbolic 3-manifold $M$, and we set $R=\operatorname{tuberad}(C)$ and $T=\operatorname{tube}(C)$. Proposition 10.1 of [3] states that

Note that

$$
\operatorname{vol} N \leq\left(\operatorname{coth}^{3} 2 R\right)\left(\operatorname{vol} M+\frac{\pi}{2} L \tanh R \tanh 2 R\right) \text {. }
$$

$$
\operatorname{vol} T=\pi L \sinh ^{2} R=\left(\frac{\pi}{2} L \tanh R\right)(2 \sinh R \cosh R)
$$

$$
=\left(\frac{\pi}{2} L \tanh R\right)(\sinh 2 R) \text {. }
$$

Thus

$$
\begin{aligned}
\operatorname{vol} N & \leq\left(\operatorname{coth}^{3} 2 R\right)\left(\operatorname{vol} M+\operatorname{vol} T \frac{\tanh 2 R}{\sinh 2 R}\right) \\
& =\left(\operatorname{coth}^{3} 2 R\right)\left(\operatorname{vol} M+\frac{\operatorname{vol} T}{\cosh 2 R}\right) .
\end{aligned}
$$


In the language of [16], the quantity $(\operatorname{vol} T) /(\operatorname{vol} M)$ is the density of a tube packing in $\mathbb{H}^{3}$. According to [16, Corollary 4.4], we have $(\operatorname{vol} T) / \operatorname{vol} M<0.91$. Hence $\operatorname{vol} N<f(x) \operatorname{vol}(M)$, where $f(x)$ is defined for $x \geq 0$ by

$$
f(x)=\left(\operatorname{coth}^{3} 2 x\right)\left(1+\frac{0.91}{\cosh 2 x}\right) .
$$

Since $f(x)$ is decreasing for $x \geq 0$, and since a direct computation shows that $f(0.5495)=3.01762 \ldots$, we have vol $N<3.0177$ vol $M$ whenever $R \geq 0.5495$.

It remains to consider the case in which $0.5495>R \geq(\log 3) / 2=0.5493 \ldots$ In this case we use [16, Theorem 4.3], which asserts that the tube-packing density $(\operatorname{vol} T) / \operatorname{vol} M$ is bounded above by $(\sinh R) g(R)$, where $g(x)$ is defined for $x>0$ by

$$
g(x)=\frac{\arcsin \frac{1}{2 \cosh r}}{\operatorname{arcsinh} \frac{\tanh r}{\sqrt{3}}} .
$$

Since $g(x)$ is clearly a decreasing function for $x>0$, and since $\sinh R$ is increasing for $x>0$, we have

$$
(\operatorname{vol} T) /(\operatorname{vol} M)<(\sinh 0.5495) g((\log 3) / 2)=0.90817 \ldots
$$

Hence vol $N<f_{1}(x) \operatorname{vol}(M)$, where $f_{1}(x)$ is defined for $x \geq 0$ by

$$
f_{1}(x)=\left(\operatorname{coth}^{3} 2 x\right)\left(1+\frac{0.90817}{\cosh 2 x}\right) \text {. }
$$

Again, $f_{1}(x)$ is decreasing for $x \geq 0$, and we see by direct computation that $f_{1}((\log 3) / 2)=3.017392 \ldots$. Hence we have vol $N<3.0174$ vol $M$ in this case.

Lemma 3.2 Suppose that $M$ is a closed, orientable hyperbolic 3-manifold such that vol $M \leq 1.22$, and that $C$ is a shortest geodesic in $M$. Set $N=\operatorname{drill}_{C}(M)$. If tuberad $(C)>(\log 3) / 2$ then the maximal cusp neighborhood in $N$ has volume less than $\pi$.

Proof We let $d(\infty)=.853276 \ldots$ denote Böröczky's lower bound [6] for the density of a horoball packing in hyperbolic space. It follows from the definition of the density of a horoball packing that the volume of a maximal cusp neighborhood in $N$ is at most $d(\infty)$ vol $N$. Lemma 3.1 gives vol $N<3.0177 \cdot 1.22<\pi / d(\infty)$, and the conclusion follows. 


\section{Filling}

As in [4], we shall say that a group is semifree if it is a free product of free abelian groups; and we shall say that a group $\Gamma$ is $k$-semifree if every subgroup of $\Gamma$ whose rank is at most $k$ is semifree. Note that $\Gamma$ is 2 -semifree if and only if every rank-2 subgroup of $\Gamma$ is either free or free abelian.

The following improved version of [4, Theorem 6.1] is made possible by more recent developments.

Theorem 4.1 Let $k \geq 2$ be an integer and let $\Phi$ be a Kleinian group which is freely generated by elements $\xi_{1}, \ldots, \xi_{k}$. Let $z$ be any point of $\mathbb{M}^{3}$ and set $d_{i}=\operatorname{dist}\left(z, \xi_{i} \cdot z\right)$ for $i=1, \ldots, k$. Then we have

$$
\sum_{i=1}^{k} \frac{1}{1+e^{d_{i}}} \leq \frac{1}{2}
$$

In particular there is some $i \in\{1, \ldots, k\}$ such that $d_{i} \geq \log (2 k-1)$.

Proof If $\Gamma$ is geometrically finite this is included in [4, Theorem 6.1]. In the general case, $\Gamma$ is topologically tame according to [1] and [7], and it then follows from [15, Theorem 1.1], or from the corresponding result for the free case in [14], that $\Gamma$ is an algebraic limit of geometrically finite groups; more precisely, there is a sequence of geometrically finite Kleinian groups $\left(\Gamma_{j}\right)_{j \geq 1}$ such that each $\Gamma_{j}$ is freely generated by elements $\xi_{1 j}, \ldots, \xi_{k j}$, and $\lim _{j \rightarrow \infty} \xi_{i j}=\xi_{i}$ for $i=1, \ldots, k$. Given any $z \in \mathbb{M}^{3}$, we set $d_{i j}=\operatorname{dist}\left(z, \xi_{i j} \cdot z\right)$ for each $j \geq 1$ and for $i=1, \ldots, k$. According to [4, Theorem 6.1], we have

$$
\sum_{i=1}^{k} \frac{1}{1+e^{d_{i j}}} \leq \frac{1}{2}
$$

for each $j \geq 1$. Taking limits as $j \rightarrow \infty$ we conclude that

$$
\sum_{i=1}^{k} \frac{1}{1+e^{d_{i}}} \leq \frac{1}{2}
$$

Let us also recall the following definition from [4, Section 8]. Let $\Gamma$ be a discrete torsion-free subgroup of $\operatorname{Isom}_{+}\left(\mathbb{H}^{3}\right)$. A positive number $\lambda$ is termed a strong Margulis number for $\Gamma$, or for the orientable hyperbolic 3-manifold $N=\mathbb{H}^{3} / \Gamma$, if whenever $\xi$ and $\eta$ are non-commuting elements of $\Gamma$, we have

$$
\frac{1}{1+e^{\operatorname{dist}(\xi \cdot z, z)}}+\frac{1}{1+e^{\operatorname{dist}(\eta \cdot z, z)}} \leq \frac{2}{1+e^{\lambda}} .
$$


The following improved version of [4, Proposition 8.4] is an immediate consequence of Theorem 4.1.

Corollary 4.2 Let $\Gamma$ be a discrete subgroup of $\operatorname{Isom}_{+}\left(\mathbb{M}^{3}\right)$. Suppose that $\Gamma$ is $2-$ semifree. Then $\log 3$ is a strong Margulis number for $\Gamma$.

Lemma 4.3 Let $N$ be a non-compact finite-volume hyperbolic 3-manifold. Suppose that $S$ is a boundary component of the compact core $\hat{N}$, and $\mathcal{H}$ is the maximal cusp neighborhood in $N$ corresponding to $S$. If infinitely many of the manifolds obtained by Dehn filling $\hat{N}$ along $S$ have 2-semifree fundamental group then $\mathcal{H}$ has volume at least $\pi$.

Proof Suppose that $\left(N_{i}\right)$ is an infinite sequence of distinct hyperbolic manifolds obtained by Dehn filling $\widehat{N}$ along $S$, and that $\pi_{1}\left(N_{i}\right)$ is 2-semifree for each $i$.

Thurston's Dehn filling theorem [5, Appendix B], implies that for each sufficiently large $i$, the manifold $N_{i}$ admits a hyperbolic metric; that the core curve of the Dehn filling $N_{i}$ of $\hat{N}$ is isotopic to a geodesic $C_{i}$ in $N_{i}$; that the length $L_{i}$ of $C_{i}$ tends to 0 as $i \rightarrow \infty$; and that the sequence of maximal tubes (tube $\left.\left(C_{i}\right)\right)_{i \geq 1}$ converges geometrically to $\mathcal{H}$. In particular

$$
\lim _{i \rightarrow \infty} \operatorname{vol}\left(\operatorname{tube}\left(C_{i}\right)\right)=\operatorname{vol} \mathcal{H} .
$$

According to Corollary 4.2, $\log 3$ is a strong Margulis number for each of the hyperbolic manifolds $N_{i}$. It therefore follows from [4, Corollary 10.5] that vol tube $\left(C_{i}\right)>V\left(L_{i}\right)$, where $V$ is an explicitly defined function such that $\lim _{x \rightarrow 0} V(x)=\pi$. In particular, this shows that

$$
\operatorname{vol} \mathcal{H} \geq \lim _{i \rightarrow \infty} V\left(L_{i}\right) \geq \pi
$$

\section{Non-exceptional manifolds, arbitrary primes}

5.1 A closed hyperbolic 3-manifold $M$ will be termed exceptional if every shortest geodesic in $M$ has tube radius at most $(\log 3) / 2$.

In this section we shall prove a result, Proposition 5.3, which gives a bound of 3 for the dimension of $H_{1}\left(M ; \mathbb{Z}_{p}\right)$ for any prime $p$ when $M$ is a non-exceptional manifold with volume at most 1.22 .

Algebraic 83 Geometric Topology, Volume 6 (2006) 
Lemma 5.2 Suppose that $M$ is a compact, irreducible, orientable 3-manifold, such that every non-cyclic abelian subgroup of $\pi_{1}(M)$ is carried by a torus component of $\partial M$. Suppose that either

(i) $\operatorname{dim} H_{1}(M ; \mathbb{Q}) \geq 3$, or

(ii) $\quad M$ is closed and $\operatorname{dim} H_{1}\left(M ; \mathbb{Z}_{p}\right) \geq 4$ for some prime $p$.

Then $\pi_{1}(M)$ is 2 -semifree.

Proof Let $X$ be any subgroup of $\pi_{1}(M)$ having rank at most 2. According to [11, Theorem VI.4.1], $X$ is free, or free abelian, or of finite index in $\pi_{1}(M)$. If $\operatorname{dim} H_{1}(M ; \mathbb{Q}) \geq 3$, it is clear that $X$ has infinite index in $\pi_{1}(M)$. If $M$ is closed and $H_{1}\left(M ; \mathbb{Z}_{p}\right) \geq 4$ for some prime $p$, then Proposition 1.1 of [17] implies that every 2-generator subgroup of $\pi_{1}(M)$ has infinite index. Thus in either case $X$ is either free or free abelian. This shows that $\pi_{1}(M)$ is 2-semifree.

Proposition 5.3 Suppose that $M$ is a closed, orientable, non-exceptional hyperbolic 3 -manifold such that vol $M \leq 1.22$. Then $H_{1}\left(M ; \mathbb{Z}_{p}\right)$ has dimension at most 3 for every prime $p$.

Proof Since $M$ is non-exceptional, there is a shortest geodesic $C$ in $M$ with $R=$ tuberad $(C)>(\log 3) / 2$. We set $N=\operatorname{drill}_{C}(M)$. Let $\mathcal{H}$ denote the maximal cusp neighborhood in $N$. Since $R>(\log 3) / 2$, Lemma 3.2 implies that vol $\mathcal{H}<\pi$.

Now assume that $\operatorname{dim} H_{1}\left(M ; \mathbb{Z}_{p}\right) \geq 4$ for some prime $p$. There is an infinite sequence $\left(M_{i}\right)$ of manifolds obtained by distinct Dehn fillings of $\hat{N}$ such that $H_{1}\left(M_{i} ; \mathbb{Z}_{p}\right)$ has dimension at least 4 for each $i$. (For example, if $(\lambda, \mu)$ is a basis for $H_{1}\left(\partial \hat{N}, \mathbb{Z}_{p}\right)$ such that $\lambda$ belongs to the kernel of the inclusion homomorphism $H_{1}\left(\partial \hat{N}, \mathbb{Z}_{p}\right) \rightarrow$ $H_{1}\left(\hat{N}, \mathbb{Z}_{p}\right)$, we may take $M_{i}$ to be obtained by the Dehn surgery corresponding to a simple closed curve in $\partial \hat{N}$ representing the homology class $\lambda+i p \mu$.) It follows from Thurston's Dehn filling theorem [5, Appendix B] that for sufficiently large $i$ the manifold $M_{i}$ is hyperbolic. Hence by case (ii) of Lemma 5.2, the fundamental group of $M_{i}$ is 2 -semifree for sufficiently large $i$. Thus Lemma 4.3 implies that vol $\mathcal{H} \geq \pi$, a contradiction.

\section{Non-exceptional manifolds, odd primes}

Proposition 6.3, which is proved in this section, gives a bound of 2 for the dimension of $H_{1}\left(M ; \mathbb{Z}_{p}\right)$ for any odd prime $p$ when $M$ is a non-exceptional manifold with volume at most 1.22 . 
Definition 6.1 Let $N$ be a connected manifold, $\star \in N$ a base point, and $Q$ a subgroup of $\pi_{1}(N, \star)$. We shall say that a connected based covering space $r:\left(N^{\prime}, \star^{\prime}\right) \rightarrow(N, \star)$ carries the subgroup $Q$ if $Q \leq r_{\sharp}\left(\pi_{1}\left(N^{\prime}, \star^{\prime}\right)\right) \leq \pi_{1}(N, \star)$

Lemma 6.2 Suppose that $\mathcal{H}$ is a maximal cusp neighborhood in a finite-volume hyperbolic 3-manifold $N$. Let $\star$ be a base point in $\mathcal{H}$, and let $P \leq \pi_{1}(N, \star)$ denote the image of $\pi_{1}(\mathcal{H}, \star)$ under inclusion. Then there is an element $\beta$ of $\pi_{1}(N, \star)$ with the following property:

$(\dagger)$ For every based covering space $r:\left(N^{\prime}, \star^{\prime}\right) \rightarrow(N, \star)$ which carries the subgroup $\langle P, \beta\rangle$ of $\pi_{1}(N, \star)$, there is a maximal cusp neighborhood $\mathcal{H}^{\prime}$ in $N^{\prime}$ which is isometric to $\mathcal{H}$.

Proof . We write $N=\mathbb{H}^{3} / \Gamma$, where $\Gamma$ is a discrete, torsion-free subgroup of Isom $\left(\mathbb{M}^{3}\right)$. Let $q: \mathbb{H}^{3} \rightarrow N$ denote the quotient map and fix a base point $\star^{\prime}$ which is mapped to $\star$ by $q$. The components of $q^{-1}(\mathcal{H})$ are horoballs. Let $B_{0}$ denote the component of $q^{-1}(\mathcal{H})$ containing $\star^{\prime}$. The stabilizer $\Gamma_{0}$ of $B_{0}$ is mapped onto the subgroup $P$ of $\pi_{1}(N, \star)$ by the natural isomorphism $\iota: \Gamma \rightarrow \pi_{1}(N, \star)$.

Since $\mathcal{H}$ is a maximal cusp, there is a component $B_{1} \neq B_{0}$ of $q^{-1}(\mathcal{H})$ such that $\overline{B_{1}} \cap \overline{B_{0}} \neq \varnothing$. We fix an element $g$ of $\Gamma$ such that $g\left(B_{0}\right)=B_{1}$, and we set $\beta=$ $\iota(g) \in \pi_{1}(N, \star)$.

To show that $\beta$ has property $(\dagger)$, we consider an arbitrary based covering space $r:\left(N^{\prime}, \star^{\prime}\right) \rightarrow(N, \star)$ which carries the subgroup $\langle P, \beta\rangle$ of $\pi_{1}(N, \star)$. We may identify $N^{\prime}$ with $\mathbb{H}^{3} / \Gamma^{\prime}$, where $\Gamma^{\prime}$ is some subgroup of $\Gamma$ containing $\left\langle\Gamma_{0}, g\right\rangle$.

Since $\Gamma_{0} \subset \Gamma^{\prime}$, the cusp neighborhood $\mathcal{H}$ lifts to a cusp neighborhood $\mathcal{H}^{\prime}$ in $N^{\prime}$. In particular $\mathcal{H}^{\prime}$ is isometric to $\mathcal{H}$. The horoballs $B_{0}$ and $B_{1}=g\left(B_{0}\right)$ are distinct components of $\left(q^{\prime}\right)^{-1}\left(\mathcal{H}^{\prime}\right)$, where $q^{\prime}: \mathbb{T}^{3} \rightarrow N^{\prime}$ denotes the quotient map. Since $g \in \Gamma^{\prime}$ and $\overline{B_{1}} \cap \overline{B_{0}} \neq \varnothing$, the cusp neighborhood $\mathcal{H}^{\prime}$ is maximal.

Proposition 6.3 Suppose that $M$ is a closed, orientable, non-exceptional hyperbolic 3 -manifold such that vol $M \leq 1.22$. Then $H_{1}\left(M ; \mathbb{Z}_{p}\right)$ has dimension at most 2 for every odd prime $p$.

Proof Since $M$ is non-exceptional, we may fix a shortest geodesic $C$ in $M$ with $R=\operatorname{tuberad}(C)>(\log 3) / 2$. We set $N=\operatorname{drill}_{C}(M)$. Let $\mathcal{H}$ denote the maximal cusp neighborhood in $N$. Since $R>(\log 3) / 2$, Lemma 3.2 implies that vol $\mathcal{H}<\pi$.

As in the statement of Lemma 6.2, we fix a base point $\star \in \mathcal{H}$, and we denote by $P \leq \pi_{1}(N, \star)$ the image of $\pi_{1}(\mathcal{H}, \star)$ under inclusion. We fix an element $\beta$ of $\pi_{1}(N, \star)$ having property $(\dagger)$ of Lemma 6.2. We set $Q=\langle P, \beta\rangle \leq \pi_{1}(N, \star)$. 
Suppose that $\operatorname{dim} H_{1}\left(M ; \mathbb{Z}_{p}\right) \geq 3$ for some prime $p$. We shall prove the proposition by showing that this assumption leads to a contradiction if $p$ is odd.

It follows from Poincare duality that the image of the inclusion homomorphism $\alpha$ : $H_{1}\left(\partial \hat{N} ; \mathbb{Z}_{p}\right) \rightarrow H_{1}\left(\hat{N} ; \mathbb{Z}_{p}\right)$ has rank 1 . Hence the image of $P$ under the natural homomorphism $\pi_{1}(N, \star) \rightarrow H_{1}\left(N ; \mathbb{Z}_{p}\right)$ has dimension 1 . It follows that the image $\bar{Q}$ of $Q$ under this homomorphism has dimension either 1 or 2 . In the case $\operatorname{dim} \bar{Q}=1$ we shall obtain a contradiction for any prime $p$. In the case $\operatorname{dim} \bar{Q}=2$ we shall obtain a contradiction for any odd prime $p$.

First consider the case $\operatorname{dim} \bar{Q}=1$. We have assumed $\operatorname{dim} H_{1}\left(M ; \mathbb{Z}_{p}\right) \geq 3$. Thus there is a $\mathbb{Z}_{p} \times \mathbb{Z}_{p}$-regular based covering space $\left(N^{\prime}, \star^{\prime}\right)$ of $(N, \star)$ which carries $Q$. By property $(\dagger)$, there is a maximal cusp neighborhood $\mathcal{H}^{\prime}$ in $N^{\prime}$ which is isometric to $\mathcal{H}$. In particular vol $\mathcal{H}^{\prime}<\pi$.

Since in particular $\left(N^{\prime}, \star^{\prime}\right)$ carries $P$, the boundary of the compact core $\widehat{N}$ lifts to $\hat{N}^{\prime}$. As $N^{\prime}$ is a $p^{2}$-fold regular covering, it follows that $\hat{N}^{\prime}$ has $p^{2} \geq 4$ boundary components.

It follows from Thurston's Dehn filling theorem [5, Appendix B] that there are infinitely many hyperbolic manifolds obtained by Dehn filling one boundary component of $\widehat{N}^{\prime}$. If $Z$ is any hyperbolic manifold obtained by such a filling, then $Z$ has at least three boundary components, and it follows from case (i) of Lemma 5.2 that $\pi_{1}(Z)$ is $2-$ semifree. It therefore follows from Lemma 4.3 that each maximal cusp neighborhood in $N^{\prime}$ has volume at least $\pi$. Since we have seen that vol $\mathcal{H}^{\prime}<\pi$, this gives the desired contradiction in the case $\operatorname{dim} \bar{Q}=1$.

It remains to consider the case in which $\operatorname{dim} \bar{Q}=2$ and the prime $p$ is odd. Since we have assumed that $\operatorname{dim} H_{1}\left(M ; \mathbb{Z}_{p}\right) \geq 3$, there is a $p$-fold cyclic based covering space $\left(N^{\prime}, \star^{\prime}\right)$ of $(N, \star)$ which carries $Q$. Since $N^{\prime}$ carries $P$, the boundary of the compact core $\hat{N}$ lifts to $\hat{N}^{\prime}$, and as $N^{\prime}$ is a $p$-fold regular covering, it follows that $\hat{N}^{\prime}$ has $p$ boundary components.

We claim that the inclusion homomorphism $\alpha^{\prime}: H_{1}\left(\partial \hat{N}^{\prime}, \mathbb{Z}_{p}\right) \rightarrow H_{1}\left(\hat{N}^{\prime}, \mathbb{Z}_{p}\right)$ is not surjective. To establish this, we consider the commutative diagram

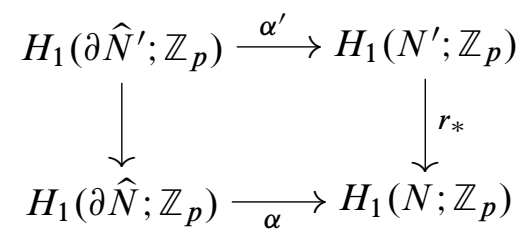

where $r: N^{\prime} \rightarrow N$ is the covering projection. Since $\left(N^{\prime}, \star^{\prime}\right)$ carries $Q$ we have $\bar{Q} \subset \operatorname{Im} r_{*}$. Hence surjectivity of $\alpha^{\prime}$ would imply $\bar{Q} \subset \operatorname{Im} \alpha$. This is impossible: we 
observed above that $\operatorname{Im} \alpha$ has rank 1 , and we are in the case $\operatorname{dim} \bar{Q}=2$. Thus $\alpha^{\prime}$ cannot be surjective.

Since $\widehat{N}^{\prime}$ has $p$ boundary components, it follows from Poincare duality that $\operatorname{dim} \operatorname{Im} \alpha^{\prime}=p \geq 3$. Since $\alpha^{\prime}$ is not surjective and $p$ is an odd prime, it follows that $\operatorname{dim} H_{1}\left(N^{\prime} ; \mathbb{Z}_{p}\right) \geq p+1 \geq 4$.

Since $\left(N^{\prime}, \star^{\prime}\right)$ carries $Q$, some subgroup $Q^{\prime}$ of $\pi_{1}\left(N^{\prime}, \star^{\prime}\right)$ is mapped isomorphically to $Q$ by $r_{\sharp}$. In particular $Q^{\prime}$ has rank at most 3 . Since $\operatorname{dim} H_{1}\left(N^{\prime} ; \mathbb{Z}_{p}\right) \geq 4$, there is a $p$-fold cyclic based covering space $\left(N^{\prime \prime}, \star^{\prime \prime}\right)$ of $\left(N^{\prime}, \star^{\prime}\right)$ which carries $Q^{\prime}$. Hence $\left(N^{\prime \prime}, \star^{\prime \prime}\right)$ is a $p^{2}$-fold (possibly irregular) based covering space of $(N, \star)$ which carries $Q$. By property $(\dagger)$, there is a maximal cusp neighborhood $\mathcal{H}^{\prime \prime}$ in $N^{\prime \prime}$ which is isometric to $\mathcal{H}$. In particular vol $\mathcal{H}^{\prime \prime}<\pi$.

Since $P \leq Q$, there is a component $T$ of $\partial \hat{N}^{\prime}$ such that $Q^{\prime}$ contains a conjugate of the image of $\pi_{1}(T)$ under the inclusion homomorphism $\pi_{1}(T) \rightarrow \pi_{1}\left(N^{\prime}\right)$. Hence $T$ lifts to the $p$-fold cyclic covering space $N^{\prime \prime}$ of $N^{\prime}$. It follows that the covering projection $r^{\prime}: N^{\prime \prime} \rightarrow N^{\prime}$ maps $p \geq 3$ components of $\left(r^{\prime}\right)^{-1}\left(\partial \hat{N}^{\prime}\right)$ to $T$. As $\hat{N}^{\prime}$ has at least three boundary components, $\widehat{N}^{\prime \prime}$ must have at least five boundary components.

Hence if $Z$ is any hyperbolic manifold obtained by Dehn filling one boundary component of $\hat{N}^{\prime \prime}$, we have $\operatorname{dim} H_{1}(Z ; \mathbb{Q}) \geq 4>3$, and it follows from case (i) of Lemma 5.2 that $\pi_{1}(Z)$ is $2-$ semifree. It therefore follows from Lemma 4.3 and Thurston's Dehn filling theorem that each maximal cusp neighborhood in $N^{\prime \prime}$ has volume at least $\pi$. Since we have seen that $\operatorname{vol} \mathcal{H}^{\prime \prime}<\pi$, we have the desired contradiction in this case as well.

\section{Exceptional manifolds}

Our treatment of exceptional manifolds begins with Proposition 7.1 below, the proof of which will largely consist of citing material from [9]. In order to state it we must first introduce some notation.

For $k=0, \ldots, 6$ we define constants $\tau_{k}$ as follows:

$$
\begin{aligned}
& \tau_{0}=0.4779 \\
& \tau_{1}=1.0756 \\
& \tau_{2}=1.0527 \\
& \tau_{3}=1.2599 \\
& \tau_{4}=1.2521 \\
& \tau_{5}=1.0239 \\
& \tau_{6}=1.0239
\end{aligned}
$$


For $k=0, \ldots, 6$ let $\mathcal{E}_{k}$ be the 2 -generator group with presentation

$$
\mathcal{E}_{k}=\left\langle x, y: r_{1, k}, r_{2, k}\right\rangle,
$$

where the relators $r_{1, k}=r_{1, k}(x, y)$ and $r_{2, k}=r_{2, k}(x, y)$ are the words listed below (in which we have set $X=x^{-1}$ and $Y=y^{-1}$ ):

$$
\begin{aligned}
& r_{1,0}=x y X y y X y x y y, \\
& r_{2,0}=X y x y x Y x y x y, \\
& r_{1,1}=X X y X Y X Y x Y X Y X y X X y y, \\
& r_{2,1}=X X y y X y x y x Y x y x y X y y, \\
& r_{1,2}=X y x y x Y x x Y x y x y X y y, \\
& r_{2,2}=X X y X X y y X y x y x y X y y, \\
& r_{1,3}=X X y x y X X y y X Y X y X Y x Y X Y x x Y X Y x Y X y X Y X y y, \\
& r_{2,3}=X X y x y X y x Y x y x Y Y x y x Y x y X y x y X X y y X Y X y y, \\
& r_{1,4}=X X y x y X y x Y x y x Y x y X y x y X X y y X Y X y X Y X y y, \\
& r_{2,4}=X X y x y X y x y X X y y X Y X y X Y x Y X Y x Y X y X Y X y y, \\
& r_{1,5}=X y X Y X y X y x y x Y x y x y, \\
& r_{2,5}=X y x y x Y x Y X Y x Y x y x y, \\
& r_{1,6}=X Y X y X Y x Y X y X Y X y x y, \\
& r_{2,6}=X Y X y x y X y x Y x y X y x y .
\end{aligned}
$$

The group $\mathcal{E}_{0}$ is the fundamental group of an arithmetic hyperbolic 3-manifold which is known as Vol3. This manifold, which was studied in [12], is described as m007 $(3,1)$ in the list given in [10], and can also be described as the manifold obtained by a $(-1,2)$ Dehn filling of the once-punctured torus bundle with monodromy $-R^{2} L$.

Proposition 7.1 Suppose that $M$ is an exceptional closed, orientable hyperbolic 3manifold which is not isometric to Vol3. Then there exists an integer $k$ with $1 \leq k \leq 6$ such that the following conditions hold:

(1) $M$ has a finite-sheeted cover $\widetilde{M}$ such that $\pi_{1}(\widetilde{M})$ is isomorphic to a quotient of $\mathcal{E}_{k}$; and

(2) there is a shortest closed geodesic $C$ in $M$ such that vol(tube $(C)) \geq \tau_{k}$.

Proof This is in large part an application of results from [9], and we begin by reviewing some material from that paper.

We begin by considering an arbitrary simple closed geodesic $C$ in a closed, orientable hyperbolic 3-manifold $M=\mathbb{H}^{3} / \Gamma$. As we pointed out in 2.2, there is a loxodromic 
isometry $f \in \Gamma$ with $A_{f} /\langle f\rangle=C$. If we set $R=\operatorname{tuberad}(C)$ and $Z=Z_{R}(f)$, it follows from the definitions that tube $(C)=Z /\langle f\rangle$, that $h(Z) \cap Z=\varnothing$ for every $h \in \Gamma-\langle f\rangle$, and that there is an element $w \in \Gamma-\langle f\rangle$ such that $w(\bar{Z}) \cap \bar{Z} \neq \varnothing$.

Let us define an ordered pair $(f, w)$ of elements of $\Gamma$ to be a GMT pair for the simple geodesic $C$ if we have (i) $A_{f} /\langle f\rangle=C$, (ii) $w \notin\langle f\rangle$, and (iii) $w(\bar{Z}) \cap \bar{Z} \neq \varnothing$. Note that since $\langle f\rangle$ must be a maximal cyclic subgroup of $\Gamma$, condition (ii) implies that the group $\langle f, w\rangle$ is non-elementary.

Set $\mathcal{Q}=\left\{(L, D, R) \in \mathbb{C}^{3}: \operatorname{Re} L, \operatorname{Re} D>0\right\}$. For any point $P=(L, D, R) \in \mathcal{Q}$ we will denote by $\left(f_{P}, w_{P}\right)$ the pair $(f, w) \in \operatorname{Isom}_{+}\left(\mathbb{H}^{3}\right) \times \operatorname{Isom}_{+}\left(\mathbb{M}^{3}\right)$, where $f, w \in$ $P G L_{2}(\mathbb{C})=\operatorname{Isom}_{+}\left(\mathbb{H}^{3}\right)$ are defined by

and

$$
f=\left[\begin{array}{cc}
e^{L / 2} & 0 \\
0 & e^{-L / 2}
\end{array}\right]
$$

$$
w=\left[\begin{array}{cc}
e^{R / 2} & 0 \\
0 & e^{-R / 2}
\end{array}\right]\left[\begin{array}{rr}
1 & 1 \\
1 & -1
\end{array}\right]\left[\begin{array}{cc}
e^{D / 2} & 0 \\
0 & e^{-D / 2}
\end{array}\right]\left[\begin{array}{rr}
1 & 1 \\
1 & -1
\end{array}\right] .
$$

With this definition, $f_{P}$ has (real) translation length $\operatorname{Re} L$, and the (minimum) distance between $A_{f}$ and $w\left(A_{f}\right)$ is $(\operatorname{Re} D) / 2$.

In [9, Section 1], it is shown that if $(f, w)$ is a GMT pair for a shortest geodesic $C$ in a closed, orientable hyperbolic 3-manifold and tuberad $(C) \leq(\log 3) / 2$, then $(f, w)$ is conjugate by some element of $\operatorname{Isom}^{+}\left(\mathbb{M}^{3}\right)$ to a pair of the form $\left(f_{P}, w_{P}\right)$ where $P \in \mathcal{Q}$ is a point such that $\exp (P) \doteq\left(e^{L}, e^{D}, e^{R}\right)$ lies in the union $X_{0} \cup \cdots \cup X_{6}$ of seven disjoint open subsets of $\mathbb{C}^{3}$ that are explicitly defined in [9, Proposition 1.28].

For every $k$ with $0 \leq k \leq 6$ and every point $P=(L, D, R)$ such that $\exp (P) \in X_{k}$, it follows from [9, Definition 1.27 and Proposition 1.28] that

(I) the isometries $r_{1, k}\left(f_{\boldsymbol{P}}, w_{\boldsymbol{P}}\right)$ and $r_{2, k}\left(f_{\boldsymbol{P}}, w_{\boldsymbol{P}}\right)$ have translation length less than $\operatorname{Re} L$;

and it follows from [9, Table 1.1] that

(II) $\pi \operatorname{Re}(L) \sinh ^{2}(\operatorname{Re}(D) / 2)>\tau_{k}$.

According to [9, Proposition 3.1], if $C$ is a shortest geodesic in a closed, orientable hyperbolic 3-manifold, and if some GMT pair for $C$ has the form $\left(f_{P}, w_{P}\right)$ for some $P$ with $\exp (P) \in X_{0}$, then $M$ is isometric to Vol3.

Now suppose that $M$ is an exceptional closed, orientable hyperbolic 3-manifold. Let us choose a shortest closed geodesic $C$ in $M$. By the definition of an exceptional manifold, $C$ has tube radius $\leq(\log 3) / 2$. Hence the facts recalled above imply that $C$ has a GMT pair of the form $\left(f_{P}, w_{P}\right)$ for some $P$ such that $\exp (P) \in X_{k}$ for some $k$ 
with $0 \leq k \leq 6$; and furthermore, that if $M$ is not isometric to Vol3, then $1 \leq k \leq 6$. We shall show that conclusions (1) and (2) hold with this choice of $k$.

For $i=1,2$ it follows from property (I) above that the element $r_{i, k}(f, \omega)$ has real translation length less than the real translation length $\operatorname{Re} L$ of $f$. Since $C$ is a shortest geodesic in $M$, it follows that the conjugacy class of $r_{i, k}(f, \omega)$ is not represented by a closed geodesic in $M$. As $M$ is closed it follows that $r_{i, k}(f, \omega)$ is the identity for $i=1,2$. Hence the subgroup of $\Gamma$ generated by $f$ and $\omega$ is isomorphic to a quotient of $\mathcal{E}_{k}$. Since we observed above that $\langle f, \omega\rangle$ is non-elementary, there is a non-abelian subgroup $Y$ of $\pi_{1}(M)$ which is isomorphic to a quotient of $\mathcal{E}_{k}$. In particular $Y$ has rank 2 , and it cannot be a free group of rank 2 since the relators $r_{1, k}$ and $r_{2, k}$ are non-trivial. Hence by [11, Theorem VI.4.1] we must have $\left|\pi_{1}(M): Y\right|<\infty$. This proves (1).

Finally, we recall that

$$
\text { vol tube }(C)=\pi(\text { length }(C)) \sinh ^{2}(\operatorname{tuberad}(C))=\pi(\operatorname{Re} L) \sinh ^{2}((\operatorname{Re} D) / 2) .
$$

Hence (2) follows from (II).

We shall also need the following slight refinement of [17, Proposition 1.1].

Proposition 7.2 Let $p$ be a prime and let $M$ be a closed 3-manifold. If $p$ is odd assume that $M$ is orientable. Let $X$ be a finitely generated subgroup of $\pi_{1}(M)$, and set $n=\operatorname{dim} H_{1}\left(X ; \mathbb{Z}_{p}\right)$. If $\operatorname{dim} H_{1}\left(M ; \mathbb{Z}_{p}\right) \geq \max (3, n+2)$, then $X$ has infinite index in $\pi_{1}(M)$. In fact, $X$ is contained in infinitely many distinct finite-index subgroups of $\pi_{1}(M)$.

Proof In this proof, as in [17, Section 1], for any group $G$ we shall denote by $G_{1}$ the subgroup of $G$ generated by all commutators and $p$-th powers, where $p$ is the prime given in the hypothesis. Since $\operatorname{dim} H_{1}\left(X ; \mathbb{Z}_{p}\right)=n$ we may write $X=E X_{1}$ for some rank- $n$ subgroup $E$ of $X$.

We first assume that $n \geq 1$. Set $\Gamma=\pi_{1}(M)$. Let $\mathcal{S}$ denote the set of all finite-index subgroups $\Delta$ of $\Gamma$ such that $\Delta \geq X$ and $\operatorname{dim} H_{1}\left(\Delta ; \mathbb{Z}_{p}\right) \geq n+2$. The hypothesis gives $\Gamma \in \mathcal{S}$, so that $\mathcal{S} \neq \varnothing$. Hence it suffices to show that every subgroup $\Delta \in \mathcal{S}$ has a proper subgroup $D$ such that $D \in \mathcal{S}$.

Any group $\Delta \in \mathcal{S}$ may be identified with $\pi_{1}(\widetilde{M})$ for some finite-sheeted covering space $\widetilde{M}$ of $M$. In particular, $\widetilde{M}$ is a closed 3-manifold, and is orientable if $p$ is odd. Since $\Delta \in \mathcal{S}$ we have $X \leq \Delta=\pi_{1}(\tilde{M})$ and $\operatorname{dim} H_{1}\left(\widetilde{M} ; \mathbb{Z}_{p}\right)=\operatorname{dim} H_{1}\left(\Delta ; \mathbb{Z}_{p}\right) \geq n+2$. Now set $D=E \Delta_{1} \leq \Delta$. Applying [17, Lemma 1.5], with $\tilde{M}$ in place of $M$, we deduce that 
$D$ is a proper, finite-index subgroup of $\Delta$, and that $\operatorname{dim} H_{1}\left(D ; \mathbb{Z}_{p}\right) \geq 2 n+1 \geq n+2$. On the other hand, since $\Delta \in \mathcal{S}$, we have $X \leq \Delta$, and hence $X=E X_{1} \leq E \Delta_{1}=D$. It now follows that $D \in \mathcal{S}$, and the proof is complete in the case $n \geq 1$.

If $n=0$ then, since $\operatorname{dim} H_{1}\left(M ; \mathbb{Z}_{p}\right) \geq 3$, there exists a finitely generated subgroup $X^{\prime} \geq X$ such that $H_{1}\left(X^{\prime} ; \mathbb{Z}_{p}\right)$ has dimension 1 . The case of the Lemma which we have already proved shows that $X^{\prime}$ has infinite index. Thus $X$ has infinite index as well.

Corollary 7.3 Let $p$ be a prime and let $M$ be a closed, orientable 3-manifold. Let $X$ be a finite-index subgroup of $\pi_{1}(M)$, and set $n=\operatorname{dim} H_{1}\left(X ; \mathbb{Z}_{p}\right)$. Then $\operatorname{dim} H_{1}\left(M ; \mathbb{Z}_{p}\right) \leq \max (2, n+1)$.

Lemma 7.4 Suppose that $M$ is an exceptional hyperbolic 3-manifold with volume at most 1.22 . Then $H_{1}\left(M ; \mathbb{Z}_{p}\right)$ has dimension at most 2 for every prime $p \neq 2,7$, and $H_{1}\left(M ; \mathbb{Z}_{2}\right)$ and $H_{1}\left(M ; \mathbb{Z}_{7}\right)$ have dimension at most 3 . Furthermore, if $M$ has volume at most 1.182 , then $H_{1}\left(M ; \mathbb{Z}_{7}\right)$ has dimension at most 2.

Proof If $M$ is isometric to Vol3 then $\pi_{1}(M)$ is generated by two elements, and the conclusions follow. For the rest of the proof we assume that $M$ is not isometric to Vol3, and we fix an integer $k$ with $1 \leq k \leq 6$ such that conditions (1) and (2) of Proposition 7.1 hold.

By condition (2) of Proposition 7.1, we may fix a shortest closed geodesic $C$ in $M$ such that $\operatorname{vol}(T) \geq \tau_{k}$, where $T=\operatorname{tube}(C)$. It follows from a result of Przeworski's [16, Corollary 4.4] on the density of cylinder packings that vol $T<0.91 \mathrm{vol} M$, and so vol $M>\tau_{k} / 0.91$. If $k=3$ we have $\tau_{k} / 0.91>1.22$, and we get a contradiction to the hypothesis. Hence $k \in\{1,2,4,5,6\}$.

Furthermore, we have $\tau_{1} / 0.91>1.182$. Hence if vol $M \leq 1.182$ then $k \in\{2,4,5,6\}$.

By condition (1) of Proposition 7.1, $\pi_{1}(M)$ has a finite-index subgroup $X$ which is isomorphic to a quotient of $\mathcal{E}_{k}$. From the defining presentations of the groups $\mathcal{E}_{1}, \mathcal{E}_{2}$, $\mathcal{E}_{4} \mathcal{E}_{5}$ and $\mathcal{E}_{6}$, we find that $H_{1}\left(\mathcal{E}_{1} ; \mathbb{Z}\right)$ is isomorphic to $\mathbb{Z}_{7} \oplus \mathbb{Z}_{7}$, that $H_{1}\left(\mathcal{E}_{2} ; \mathbb{Z}\right)$ and $H_{1}\left(\mathcal{E}_{4} ; \mathbb{Z}\right)$ are isomorphic to $\mathbb{Z}_{4} \oplus \mathbb{Z}_{12}$, while $H_{1}\left(\mathcal{E}_{5} ; \mathbb{Z}\right)$ and $H_{1}\left(\mathcal{E}_{6} ; \mathbb{Z}\right)$ are isomorphic to $\mathbb{Z}_{4} \oplus \mathbb{Z}_{4}$. (One can check that the two groups $\mathcal{E}_{5}$ and $\mathcal{E}_{6}$ are isomorphic to each other.) In particular, since $k \in\{1,2,4,5,6\}$ we have $\operatorname{dim} H_{1}\left(\mathcal{E}_{k} ; \mathbb{Z}_{p}\right) \leq 1$ for any prime $p \neq 2,7$, and $\operatorname{dim} H_{1}\left(\mathcal{E}_{k} ; \mathbb{Z}_{p}\right) \leq 2$ for $p=2$ or 7 . As $X$ is isomorphic to a quotient of $\mathcal{E}_{k}$, it follows that $\operatorname{dim} H_{1}\left(X ; \mathbb{Z}_{p}\right) \leq 1$ for any prime $p \neq 2,7$, and $\operatorname{dim} H_{1}\left(X ; \mathbb{Z}_{p}\right) \leq 2$ for $p=2$ or 7 . Hence by Corollary 7.3 , we have $\operatorname{dim} H_{1}\left(M ; \mathbb{Z}_{p}\right) \leq 2$ for $p \neq 2,7$, and $\operatorname{dim} H_{1}\left(M ; \mathbb{Z}_{p}\right) \leq 3$ for $p=2,7$. 
It remains to prove that if vol $M \leq 1.182$ then $\operatorname{dim} H_{1}\left(M ; \mathbb{Z}_{7}\right) \leq 2$. We have observed that in this case $k \in\{2,4,5,6\}$. By the list of isomorphism types of the $H_{1}\left(\mathcal{E}_{k} ; \mathbb{Z}\right)$ given above, it follows that $\operatorname{dim} H_{1}\left(\mathcal{E}_{k} ; \mathbb{Z}_{7}\right)=0<1$. Hence in this case the argument given above for $p \neq 2,7$ goes through in exactly the same way to show that $\operatorname{dim} H_{1}\left(M ; \mathbb{Z}_{7}\right) \leq 2$.

Proof of Theorem 1.1 For the case in which $M$ is non-exceptional, the theorem is an immediate consequence of Propositions 5.3 and 6.3. For the case in which $M$ is exceptional, the assertions of the theorem are equivalent to those of Lemma 7.4.

\section{References}

[1] I Agol, Tameness of hyperbolic 3-manifolds arXiv:math.GT/0405568

[2] I Agol, Volume change under drilling, Geom. Topol. 6 (2002) 905-916 MR1943385

[3] I Agol, N M Dunfield, PA Storm, W P Thurston, Lower bounds on volumes of hyperbolic Haken 3-manifolds arXiv:math.DG/0506338

[4] J W Anderson, R D Canary, M Culler, P B Shalen, Free Kleinian groups and volumes of hyperbolic 3-manifolds, J. Differential Geom. 43 (1996) 738-782 MR1412683

[5] M Boileau, J Porti, Geometrization of 3-orbifolds of cyclic type, Appendix A by M Heusener and J Porti, Astérisque 272 (2001) MR1844891

[6] K Böröczky, Packing of spheres in spaces of constant curvature, Acta Math. Acad. Sci. Hungar. 32 (1978) 243-261 MR512399

[7] D Calegari, D Gabai, Shrinkwrapping and the taming of hyperbolic 3-manifolds, J. Amer. Math. Soc. 19 (2006) 385-446 MR2188131

[8] M Culler, P B Shalen, Paradoxical decompositions, 2-generator Kleinian groups, and volumes of hyperbolic 3-manifolds, J. Amer. Math. Soc. 5 (1992) 231-288 MR1135928

[9] D Gabai, G R Meyerhoff, N Thurston, Homotopy hyperbolic 3-manifolds are hyperbolic, Ann. of Math. (2) 157 (2003) 335-431 MR1973051

[10] C Hodgson, J Weeks, Downloadable text file Available at http:// www. geometrygames.org/SnapPea/

[11] W H Jaco, P B Shalen, Seifert fibered spaces in 3-manifolds, Mem. Amer. Math. Soc. 21 (1979) viii+192 MR539411

[12] K N Jones, A W Reid, Vol3 and other exceptional hyperbolic 3-manifolds, Proc. Amer. Math. Soc. 129 (2001) 2175-2185 MR1825931

[13] S Kojima, Deformations of hyperbolic 3-cone-manifolds, J. Differential Geom. 49 (1998) 469-516 MR1669649 
[14] H Namazi, J Souto, Nonrealizability in handlebodies and ending laminations in preparation

[15] K Ohshika, Realizing end invariants by limits of minimally parabolic, geometrically finite groups arXiv:math.GT/0504546

[16] A Przeworski, A universal upper bound on density of tube packings in hyperbolic space, J. Differential Geom. 72 (2006) 113-127 MR2215457

[17] P B Shalen, $\mathbf{P}$ Wagreich, Growth rates, $Z_{p}$-homology, and volumes of hyperbolic 3-manifolds, Trans. Amer. Math. Soc. 331 (1992) 895-917 MR1156298

Department of Mathematics, Statistics, and Computer Science (M/C 249)

University of Illinois at Chicago, 851 S Morgan St, Chicago, IL 60607-7045, USA

agol@math.uic.edu, culler@math.uic.edu, shalen@math.uic.edu

Received: 14 July 2006

Algebraic 83 Geometric Topology, Volume 6 (2006) 